\title{
On location-based services build on the close spatial position of mobile devices
}

\author{
Dmitry Namiot, \\ Lomonosov Moscow State University, \\ GSP-1, Leninskie Gory, Moscow, 119991 \\ Russia
}

\begin{abstract}
This article focuses on architectural models of location-based services. The paper discusses the model of spatial network proximity, within which the classical architecture of services using location information, based on the use of geo-coordinates data provided by users, is replaced by some distributed cyber-physical system. Within the network proximity model, geo-computation is replaced by the direct proximity definitions. And this very proximity measurement is based on determining the availability (visibility) of the signals of the wireless network nodes. This article discusses how to build new service models using location information.
\end{abstract}

Keywords—location-based services, network proximity.

\section{INTRODUCTION}

L OCATION based services (LBS) are among the most sought after by mobile users. In addition to such services, analytics (analytical reports) related to the analysis of information about user movements are also in demand. As confirmation of this fact, one can cite, for example, the following figures:

- the volume of the location-based services market was \$ 28.95 billion in 2019 , and is expected to reach $\$ 183.81$ billion by 2027 [1];

- Search query "proximity services" on Google Scholar shows more than 41,000 mentions in patents since 2016.

If we look at the architecture of LBS, then this element has not changed in many years. LBS systems are a classic example of a client-service system, when a client (application) receives location information, in the overwhelming majority of cases, using global positioning systems (GPS) and transmits this information to the service. The service, having received the coordinates, selects the closest service - fig. 1 .

The innovations in this area concerned only the server-side. For example, new ways of storing geo-positional information [2], geo-extensions for databases [3], etc.

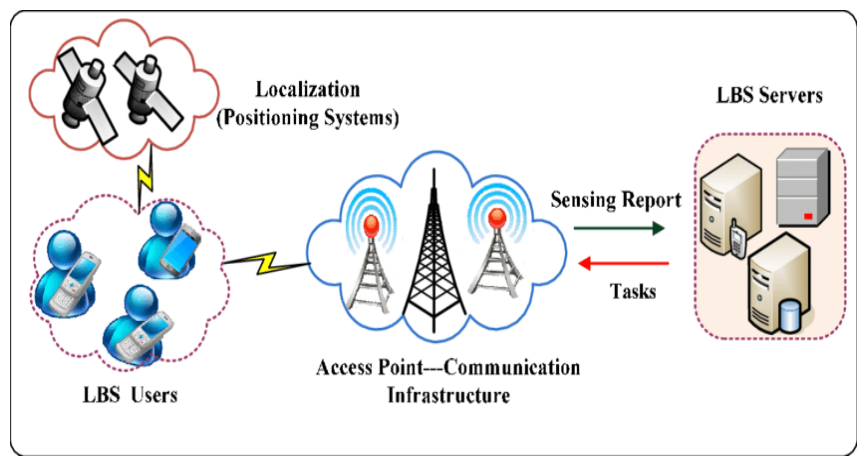

Fig. 1. LBS architecture [2]

It is a simple model that builds on the ubiquity of smartphones that support global positioning systems (GPS). The client application receives the current coordinates (this is programmatically standardized, for example, W3C) and can provide them to the service. This model was not always like this. For example, back in the early 2000s, a client application could receive location information only from a telecom operator (later - also from a mashup, which emulated to some extent the operator's work, providing information about the location of the operator's base stations), but the proliferation of smartphones brought everything together eventually to the use of GPS and a simple client-server system.

In general, LBS applications are typical context-sensitive applications, the current location is always part of the context [4]. 
But, unlike other contextual data, location information takes a separate place. This data is directly related to the privacy of mobile users (subscribers). The problem of maintaining privacy is a hot problem for most users [5, 6]. Using the above-mentioned method of assessing scientific relevance, more than 310,000 links after 2016 can be mentioned for the search term "location sharing privacy" at scholar.google.com.

It should be noted that within the existing model, there is no way to avoid transmitting location information to the provider. It is possible, for example, to coarse coordinates in some way (for example, spatial cloaking [7]), but in principle, the service provider will be able to track the use of the service. It is possible to use other protocols for the "safe" exchange of positioning information [8], but they also do not change the basic scheme.

The exact same scheme remains in effect for social networks, where a special form of check-in was introduced. This mark was introduced as a special message on a social network that contained location information. The idea is to search for other users (other content) with similar location stamps inside or even outside the social circle. In this case, again, it is essential to transfer information about the location to a third-party provider (social network).

It should be noted that in most LBS services, the actual information about the coordinates is not needed. Coordinates are used as keys to finding data. Most of the services provide services (information) in the vicinity of some point. Most often, this is either the current location of the client ("find something near me"), or some future (possible) location ("find something near cafe C").

From a formal point of view, describing such semantics on GeoSparql [9], for example, this means that most services are based on the execution (interpretation) of the nearby function

Here's what a rule might look like to interpret this function:

$$
\begin{aligned}
\text { ? A geo: nearby? B: - A? geom? g1 } & \text { B? geom? g2 } \\
& \text { filter (distance } \\
\text { g1, ? g2) <50) } &
\end{aligned}
$$

the geometric characteristics of objects $A$ and $B$ are taken and it is checked that the distance between them is less than a certain limit (50 meters in this example). And, accordingly, in the query where specific objects are operated: ? Cafe geo: nearby? Street, the function geo:nearby will be interpreted according to the above rule.

Based on this, the idea appeared to change the architecture (implementation model) of LBS applications to directly calculate this spatial proximity, without resorting to geocoordinates. This model is called the network spatial proximity model. And this article is devoted to the consideration of new models of LBS applications (services).
The rest of the article is structured as follows. Section II deals with the network spatial proximity model and its characteristics that provide new service models. Section III deals with one class of new models of LBS applications, the implementation of which is precisely based on the use of network spatial proximity.

\section{ON NETWORK PROXIMITY MODELS}

Within the framework of this approach, to determine the proximity of mobile devices (as mentioned above, this is intended to replace the work with geo-coordinates), it is proposed to use the physically limited propagation of the signal of wireless networks.

From a software point of view, discovering a wireless device is getting its advertising information. This is how, for example, searching for neighbors in wireless networks works. Neighbor Search is the definition of all nodes in the network with which a given node can communicate directly. Obviously, nodes must use (transmit) some identification during the discovery process. And the idea of network spatial proximity is precisely to use this identification information (or some addons for this information) to transfer user data.

The limited area of the signal propagation of wireless networks is precisely the basis for determining proximity. It does not use connections in any form, and no attempt is made to estimate the location. This makes it possible to use not only any existing wireless network nodes (Wi-Fi access points, Bluetooth tags, etc.) to determine network spatial proximity, but also create such nodes specifically for proximity detection tasks. In the latter case, you can arbitrarily set the identification of such nodes (their advertising presentation), as well as update it dynamically [10]. For example, a Bluetooth Low Energy device in advertising mode (advertiser) periodically transmits advertising information (Fig. 2) in three presentation channels and listens, waiting for a response from other devices.

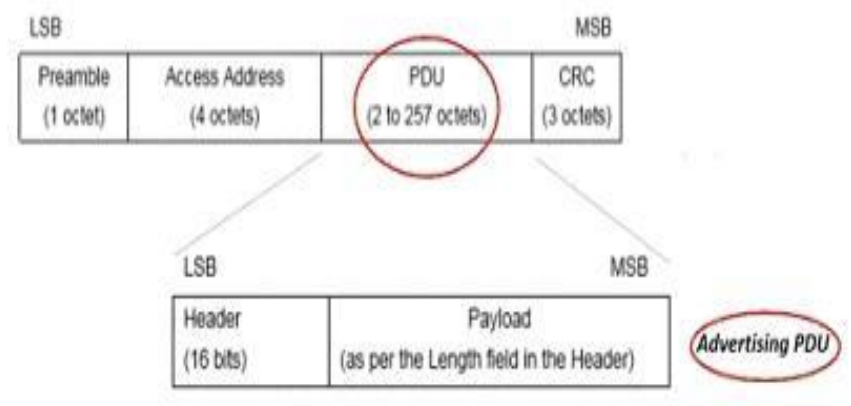

Fig. 2. Bluetooth Promotional Package [11]

On the other hand, a device in scanning mode (called a scanner) periodically scans presentation channels (advertising channels) and listens to advertising information from other devices. Promoters differ only in that they can only respond to 
specific types of advertising packages. The payload in Figure 2 just allows you to transfer user information in an advertising package without communication between devices.

This work uses not only its format for Bluetooth LE as an advertising presentation but also, for example, the SSID for the Wi-Fi node. It is also broadcast information. And since no connections are assumed for hosts in the used model (architecture used), there are no restrictions for specifying the content of such mailings (they are now intended not for endusers, but for applications).

Thus, in the network spatial proximity model, geocoordinates are replaced by the identification of wireless network nodes. Accordingly, the proximity check, classically presented as a comparison of coordinates, in the new model is presented as a check of the visibility (availability) of certain nodes of wireless networks. And this check, in turn, consists of fixing the receipt of advertising (identification) of the wireless node. In addition to advertising a wireless node, which fixes the fact of its availability (visibility), its other available characteristics can also be taken into account. For example, signal strength (RSSI), direction (for Bluetooth 5), etc. These characteristics can be taken into account in the conditions for the provision of LBS.

\section{ClOSE SPATIAL POSITION IN LOCATION-BASED SERVICES}

Determination of available network nodes (this is also often called network fingerprint) can be done directly in the client's mobile application. Verification of the terms of service depending on the location can then also be performed directly on the client-side, if its application includes a copy of the database with information about services, or on the service side, where the client contacts, transferring data about the available network environment (network fingerprint).

Formally, the definition of proximity can be represented as some predicate

$$
P\left(N_{1}, N_{2}, \ldots N_{k}\right) \rightarrow\{A, C\}
$$

Where $N_{i}$ is the description of the visible (accessible) node. This description includes, as indicated in section II, all available (measurable) characteristics of a particular network node, including information about its advertising presentation. The logical gates in this predicate can describe the conditions imposed on these characteristics. For example, describe possible boundaries for the strength of the RSSI signal, or simply check the availability of a site with a given advertising presentation:

$$
\begin{aligned}
& \text { N.RSSI < -70 } \\
& \exists N . S S I D=" L o b b y "
\end{aligned}
$$

The logical combination ( $A N D, O R, N O T$ ) of such elements forms a predicate, which is executed to check the proximity to the specified reference nodes (node) of the wireless network. $A$ and $C$ in the above formula are possible location-based actions and location-based content, respectively.

The calculation of such predicates is the test of network proximity. A set of these types of rules can also underlie a fuzzy logic system for selecting rules from several suitable ones. In practice, in a mobile application (for example, an application from a retail retailer), information about available (visible) network nodes is checked, on the basis of which the application can understand whether the user is currently in one of the shopping malls (halls) and in which one of them, or the application is launched outside the walls of the shopping mall.

In the present work, a model (architecture) is considered that describes LBS applications based on the proximity of several mobile devices to each other.

The system has the following parties:

- Provider (s) of location-specific content (services)

- Operator (provider) of proximity determination (may coincide with service providers)

- Service customer operating with several mobile devices (support devices)

- Users (mobile clients).

The scheme of operation of this kind of system is as follows.

1. The customer of the service associates the provision of his content (performance of any actions) with the proximity of the client's mobile devices and the mobile devices selected by him (the customer) as dedicated (reference) mobile devices.

2. Supporting devices periodically inform the provider operator of proximity determination information about the network environment available to him (visible network nodes, fingerprints).

3. Client devices (mobile applications) for requesting LBS services transmit information about the available (visible) network environment to the proximity provider.

4. The provider compares client information and data from reference devices. On a match, the content associated with the corresponding reference device is returned.

This is illustrated in Figure 3. The reference device then acts as a tag. Moreover, such a tag is automatically mobile (movable tag).

Figure 3 shows the reference device A, client device B, and a shared (accessible to both devices) Wi-Fi access point. The latter will determine the general network environment, which means the mutual proximity of devices A and B.

In this form, the proposed schema for LBS applications has a clear business view (business model). The customer of the service receives support from the provider in terms of determining the proximity of arbitrary client devices to the ordered reference devices. The customer places (moves) these supporting devices (mobile phones, mobile terminals) in an 
arbitrary manner inside their own premises (territory, vehicles, etc.). The mobile application on the reference device can be the same for all users. All that is required of him is to periodically send information about the network environment to the provider.

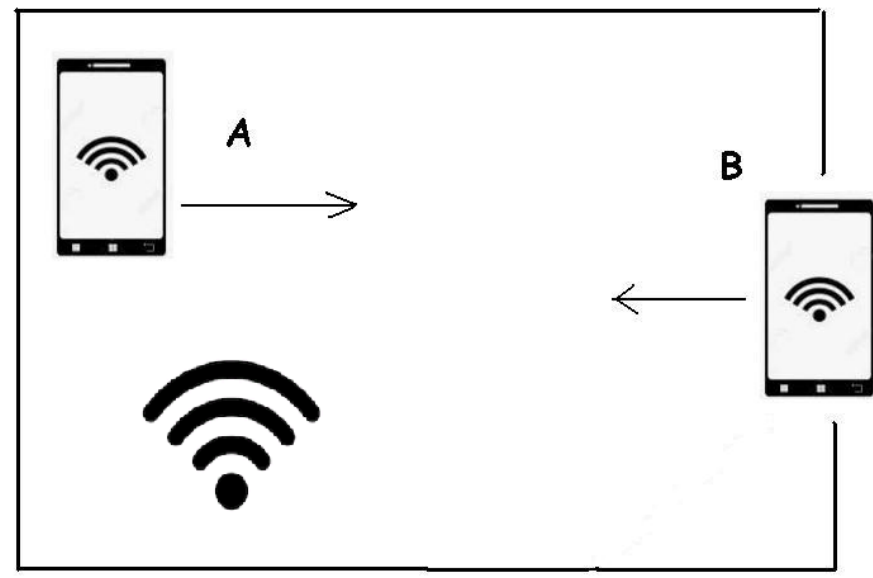

Fig. 3. On the definition of the general network environment.

The client mobile application must also send information about the network environment to the provider. This sending is a confirmation of the desire to receive the service (s) depending on the location. You can replace that conceptually such a submission corresponds to the concept of check-in in social networks. In social networks, this is a record (message) with information about location, designed to provide information from the network (social circle), taking into account the specified location. In the described model, this is a signal of the same.

Within this business model, the service provider can evaluate its performance in a variety of ways. It can be some kind of uniform (flat price), it can depend on the number of devices. Since in such a model all checks for the provision of services go through the provider, billing schemes (tariffs) are possible taking into account the number of calls, their intensity, as well as the time it takes to execute requests.

This is a new architecture for LBS applications and a new business model for service providers.

Obviously, a proximity provider can also store activity and content information (that is, the actual location-specific data). Particularly interesting is the scheme in which a telecommunications operator will act as such a proximity computing provider. The LBS scheme is now fully decentralized. Clients determine their location on their own, operators are not represented in LBS business models. In the proposed scheme, the mobile operator receives the natural role of a provider for calculating proximity (in fact, for an alternative form of geo-computing).

The use of the advertising presentation of the wireless node leads in this case to another service model. We are talking about broadcasting any text information. There are several important points to note. In such services, there is no need to organize the coordinated work of the transmitter and recipients of the information. It is enough just to repeat the entire text several times. Then, any receiver in the middle of a transmission (which appeared next to the transmitter after the broadcast started) would receive and display only a portion of the information in the first receive cycle, and then receive and display all of the text. This will be a complete imitation of the information tape on the scoreboard.

To transmit long texts, we can split them into shorter packets (strings), the last character of which indicates the need to concatenate the next packet upon receipt. Thus, the recipient can assemble the individual parts into a complete text.

Secondly, there can be multiple transmitters. The receivers will distinguish them by the addresses that are present in the advertising packages.

In addition, it is obvious that we can support the presentation of some structured information in the text. For example, when displaying the received text, it will automatically highlight phone numbers, email addresses, etc. This will allow us to display the data most convenient for later use (the ability to directly call the number, etc.).

This scheme eliminates the need for separate storage of information about geo-referenced content. All necessary information will be directly broadcast by the reference devices. And the recipients of such data can be all mobile clients that are close to the reference device.

From the point of view of the business model, this option opens up new forms of presentation (presentation) of advertising content, which can be dynamic (support devices can, of course, change their mailings) and localized (available only in a limited area).

\section{CONCLUSION}

In this article, we described a new model for services using location information. It is based on the use of architectural solutions of the network spatial proximity model. This model compares the characteristics of the network environment of user (client) devices and some dedicated mobile devices that play the role of mobile tags. The proximity of the client mobile device and some reference device is the basis for the provision of the service.

\section{ACKNOWLEDGMENT}

The author is grateful to the staff of the Laboratory of Open Information Technologies of the Faculty of CMC, Lomonosov Moscow State University for useful discussions and criticism at seminars that contributed to the improvement of this work. 


\section{REFERENCES}

[1] Location-based service market https://www.alliedmarketresearch.com/location-basedservices-market. Retrieved: Dec, 2020

[2] Abdalla, Rifaat. "Mobile GIS and location-based services (LBS)." Introduction to Geospatial Information and Communication Technology (GeoICT). Springer, Cham, 2016. 83-103.

[3] Allheeib, Nasser, et al. "Density-based reverse nearest neighbourhood search in spatial databases." Journal of Ambient Intelligence and Humanized Computing (2018): $1-12$.

[4] Namiot, Dmitry, and Manfred Sneps-Sneppe. "Contextaware data discovery." 2012 16th International Conference on Intelligence in Next Generation Networks. IEEE, 2012.

[5] Xie, Jierui, Bart Piet Knijnenburg, and Hongxia Jin. "Location sharing privacy preference: analysis and personalized recommendation." Proceedings of the 19th international conference on Intelligent User Interfaces. 2014.

[6] Tsai, Janice Y., et al. "Location-sharing technologies: Privacy risks and controls." Isjlp 6 (2010): 119.

[7] Niu, Ben, et al. "A fine-grained spatial cloaking scheme for privacy-aware users in location-based services." 2014 23rd international conference on computer Communication and networks (ICCCN). IEEE, 2014.

[8] Namiot, Dmitry, and Manfred Sneps-Sneppe. "Where are they now-safe location sharing." Internet of Things, Smart Spaces, and Next Generation Networking. Springer, Berlin, Heidelberg, 2012. 63-74.

[9] Battle, Robert, and Dave Kolas. "Geosparql: enabling a geospatial semantic web." Semantic Web Journal 3.4 (2011): 355-370.

[10]Namiot, Dmitry, and Manfred Sneps-Sneppe. "On proximity-based information delivery." International Conference on Distributed Computer and Communication Networks. Springer, Cham, 2018.

[11]Bluetooth Low Energy - It starts with Advertising http://blog.bluetooth.com/bluetooth-low-energy-it-startswith-advertising Retrieved: Dec, 2020

\section{Creative Commons Attribution License $\mathbf{4 . 0}$ (Attribution 4.0 International, CC BY 4.0)}

This article is published under the terms of the Creative Commons Attribution License 4.0 https://creativecommons.org/licenses/by/4.0/deed.en US 